\title{
Representaciones sociales y atribuciones del alcohol y subvaloración personal en canciones de la sierra peruana
}

\author{
Fredy Fernando Rodríguez Canales \\ fredy.rodríguez@unh.edu.pe \\ Doctor en psicología \\ Responsable de la Vigilancia Epidemiológica en Salud Mental \\ en la Dirección Regional de Salud Huancavelica. \\ Docente invitado en posgrado en \\ Universidad Nacional de Huancavelica \\ Huancavelica - Perú \\ Antonieta del Pilar Uriol Alva \\ antonieta.uriol@unh.edu.pe \\ Psicóloga, Doctora en educación \\ Docente; Universidad Nacional de Huancavelica \\ Huancavelica - Perú
}

\section{RESUMEN}

El artículo trata sobre las representaciones sociales y atribuciones respecto al consumo de alcohol y la sub valoración personal en expresión de la subjetividad en la población de la sierra peruana derivado de un estudio de tipo básico en un nivel hermenéutico - documental de análisis de contenido de los mensajes de una antología de ciento seis (106) canciones procedentes de esta región. En las canciones analizadas se identifican representaciones sociales donde se interpreta al licor como remedio de penas y sufrimientos, autoconcepto negativo con resignación al abandono y sufrimiento, evasión con ideas de autoeliminación, demandas de compañía y afecto, y objetivos de vida imprecisos. Como atribuciones se identifican y analizan el resentimiento, frustraciones afectivo-emocionales, sufrimiento y desamparo, enamoramiento, recuerdos y desconsuelo, y la dependencia alcohólica. Se reflexionan acerca de estas expresiones en integración de cuatro paradigmas del campo de la psicología; las representaciones sociales de Jodelet y Moscovici, las atribuciones de Heider, el aprendizaje social de Bandura y Walters, y la indefensión aprendida de Seligman y Rosellini.

Palabras clave: representaciones sociales; atribuciones, consumo de alcohol, subvaloración personal 


\title{
Social representations and attributions of alcohol and personal undervaluation in lyrics of the peruvian sierra
}

\begin{abstract}
The article deals with social representations and attributions regarding alcohol consumption and personal undervaluation in expression of subjectivity in the population of the Peruvian highlands derived from a basic study at a hermeneutic level - documentary content analysis of the messages from an anthology of one hundred and six (106) in lyrics from this region. In the songs analyzed, social representations are identified where liquor is interpreted as a remedy for pain and suffering, negative self-concept with resignation to abandonment and suffering, avoidance with ideas of self-elimination, demands for company and affection, and imprecise life goals. Resentment, affective-emotional frustrations, suffering and helplessness, infatuation, memories and grief, and alcohol dependence are identified and analyzed as attributions. These expressions are reflected on in integration of four paradigms from the field of psychology; Jodelet and Moscovici's social representations, Heider's attributions, Bandura and Walters' social learning, and Seligman and Rosellini's learned helplessness.
\end{abstract}

Keywords: social representations; attributions, alcohol consumption, personal undervaluation

Artículo recibido: 30 noviembre. 2021 Aceptado para publicación: 29 diciembre 2021 Correspondencia: fredy.rodríguez@unh.edu.pe Conflictos de Interés: Ninguna que declarar 


\section{INTRODUCCIÓN}

En el Perú, la región de la sierra que alberga alrededor del $28.1 \%$ de la población del país (INEI, 2017), es considerada el espacio geográfico de difícil hábitat para el ser humano. En la actualidad comprende a los pueblos con la mayor incidencia de pobreza que en promedio afecta al $37.4 \%$ de su población, siendo esta incidencia aún mayor en la Sierra rural que alcanza al 50.4\% (INEI, 2021), identificados como "los más pobres del Perú". Los avances en las Tecnologías de la Información y las Comunicaciones (TIC), se catalogan como una revolución tecnológica que vive actualmente la humanidad cuyo impacto en la sociedad se caracterizan por: la generalización del uso de las tecnologías, las redes de comunicación, el rápido desenvolvimiento tecnológico y científico, y la globalización de la información (Díaz, Pérez y Florido, 2011). Son la radio, la televisión, los productos video-audio-fono magnéticos en general, el internet y las redes sociales los elementos comunicacionales de gran alcance y manipulación que influencian en la formación y expresión de los estilos de vida de las personas, como lo es en particular en los pueblos de la sierra peruana donde, gracias a estos medios las expresiones folclóricas han alcanzado elevados patrones de difusión y preferencias, siendo una de ellas el canto en sus diversos ritmos, géneros y estilos.

El estudio tuvo como objetivo central identificar las representaciones sociales y atribuciones para el consumo de alcohol y la subvaloración personal expresadas en las canciones procedentes de la sierra peruana, centrándose en el análisis de las canciones comúnmente identificadas como pertenecientes a la sierra peruana, tales como: huaynos, huaylarshs, mulizas, pasacalles, yaravíes, chuscadas, carnavales, qachuas, etc., en la perspectiva de aproximarse al entendimiento de las características psicosociales de un sector de la población peruana, en cuanto a representaciones sociales y atribuciones para el consumo de bebidas alcohólicas y la sub valoración personal expresadas en el discurso de una antología de 106 canciones sobre la base de cuatro paradigmas de la psicología social: las representaciones sociales, las atribuciones, el aprendizaje social, y la indefensión aprendida. Las canciones expresan el sentir del pueblo, sus vivencias y deseos más profundos (Robayo, 2015), donde las representaciones sociales son formas de pensamiento práctico orientados hacia la comunicación, la comprensión y el dominio del entorno social, material e ideal. (Jodelet,1993). Para Moscovici (2002), implica al conjunto de conceptos y enunciados explicativos ocurridos en la interacción social cotidiana. Cual sentido común, 
corresponden a los mitos y creencias tradicionales basados en constructos cognitivos compartidos.

Por otro lado, una atribución es el proceso en virtud al cual las personas adjudican o infieren causas a determinados acontecimientos o comportamientos (Heider, 1958). Las atribuciones son argumentos o explicaciones acerca del por qué ocurrieron ciertos hechos o por qué algunas personas se comportaron en cierta forma (Hirt y McCrea, 2002). En otras palabras, atribuir es explicar las causas de determinados hechos, acontecimientos o conductas.

Ahora bien, una explicación teórica acerca de cómo determinadas expresiones o ideas se instauran de manera compartida o colectiva la podemos encontrar en el paradigma del aprendizaje social o vicario, que fue postulado por Bandura y Walters (1990). Bajo este enfoque, existen determinados comportamientos personales o colectivizados (por ejemplo, las representaciones sociales y las atribuciones) que son asimilados por la persona. Los espacios donde comúnmente se moldean y refuerzan dichos comportamientos son en el hogar, la escuela, los amigos o la comunidad que son los entornos sociales de mayor influencia donde yacen los referentes o modelos de especial significación afectiva de las personas.

Por su parte, el paradigma del desamparo o la indefensión aprendida, de acuerdo a Rosellini y Seligman (1975), explican cómo las personas adquieren una predisposición para darse por vencidas y ser incapaces de enfrentar situaciones de adversidad. Esta predisposición se da como resultado del convencimiento y la creencia de que, sea cual sea el emprendimiento u objetivo, no va a funcionar. A su vez, esta creencia surge como consecuencia de reiteradas vivencias de frustración, las cuales llegan convertirse finalmente en estados de resignación y pasividad.

En el estudio de las representaciones sociales y las atribuciones, influenciada en cierta forma por el proceso de globalización, se toma menos en cuenta la dimensión de la subjetividad, que involucra aspectos como la afectividad, emocionalidad, espiritualidad y la salud mental de las personas. Se olvida que estos son factores de gran influencia que están intrínsecamente ligados al desarrollo y la calidad de vida. Por tanto, se hace necesario analizar los aspectos de orden psicosocial como; la moral, la ética, los valores, actitudes, habilidades sociales, espiritualidad o el sentido de trascendencia cuyo fomento y enseñanza no sólo tienen que ver con la educación formal o institucionalizada, sino también con la 
educación informal o cotidiana, que se expresa en manifestaciones socio culturales que advertimos, algunos son de riesgo, tal como se pone de manifiesto en algunas canciones típicas y de arraigo popular en estas poblaciones, las cuales directa o indirectamente hacen elogio a estilos de vida no saludables, tales como el consumo irresponsable y sin control de bebidas alcohólicas, promoviendo incluso, directa o indirectamente pobreza con respecto a habilidades sociales para hacer frente a la vida bajo una concepción de fatalismo, desesperanza y subvaloración personal.

Muchas de estas expresiones nos aproximan a entender el statu quo del desarrollo humano y de la subjetividad que poseen algunos pobladores de la sierra peruana, así como a entender sus proyecciones y perspectivas de progreso, las cuales en gran parte provienen de un aprendizaje social. Resulta pues ineludible y necesario el entendimiento de las representaciones sociales y atribuciones que subyacen en estas poblaciones, en tanto que su estudio permitirá que las estrategias orientadas a promover actitudes proactivas en favor del desarrollo integral tengan un enfoque más adecuado, y consideren nuevas propuestas de acción. Así mismo, nos demuestra la importancia de facilitar la difusión de discursos alternativos positivos, orientados a promover en la persona y las colectividades la capacidad de gestionar su bienestar y su progreso.

En consecuencia, se hace necesario realizar un análisis sistemático de las características y orientación de los mensajes canticos de la sierra peruana, tales como las canciones que hacen alusión al consumo de alcohol y la subvaloración personal. Estas expresiones culturales son una exteriorización de la subjetividad personal y social de las poblaciones, las cuales se fundamentan a partir de diversas representaciones sociales y atribuciones, cuyo estudio brinda aproximación al entendimiento de los sentimientos, creencias y explicaciones que las colectividades le dan a los acontecimientos que en la cotidianidad la vivencian.

Desde la perspectiva de las ciencias sociales, la riqueza y variedad de las expresiones culturales como las canciones, son las que definen sustancialmente parte de la identidad de los pueblos y se genera una necesidad de preservarlas, rescatarlas, revalorarlas e incluso difundirlas; sin embargo, desde la posición de las ciencias de la salud y la psicología, es importante diferenciar a aquellas expresiones merecedoras de valoración y difusión, por ser fortalezas y elementos protectores para la población, y que contribuyen a hacerlos menos vulnerables, de aquellas expresiones que refuerzan ideas de debilidad y desventaja, 
en tanto se orientan no a fines constructivos y de optimismo, sino a la irresponsabilidad, la desesperanza o al fracaso, las cuales, al ser interiorizadas y colectivizadas como representaciones sociales y atribuciones limitan la atención de sus necesidades y demandas. Un ejemplo claro de estas últimas lo podemos encontrar en un grupo de canciones cuyos contenidos aluden al consumo de alcohol y la subvaloración.

\section{ESTRATEGIAS METODOLÓGICAS}

El estudio fue de tipo básico en un nivel hermenéutico - documental. Buscó comprender las representaciones sociales y sus atribuciones para el consumo de alcohol y la sub valoración personal como fenómeno psicosocial en base al análisis de contenido de los mensajes de una antología de canciones procedentes de la sierra peruana.

Se revisaron 531 canciones, contenidas en CDs que fueron adquiridos en puestos de venta ambulatorios (en adelante "grupo de canciones de la sierra en general") de los cuales, por selección intencionada a modo de tamizaje a cargo de un equipo de cuatro profesionales de las ciencias sociales: 01 antropóloga, 01 educador, 01 socióloga y 01 psicólogo, se identificaron 106 canciones que fueron las unidades de análisis, (en adelante "grupo de canciones analizadas"). El criterio básico para la identificación de este grupo de canciones que fueron objeto de análisis fue, que sus estrofas hagan alusión a las bebidas alcohólicas, su consumo o expresen un sentido de subvaloración personal.

Con el objetivo de elaborar el instrumento de análisis que defina las categorías de representación social y atribuciones, se tomaron un grupo de seis (6) canciones representativas. Para ello, se solicitó el apoyo de 06 profesionales de las ciencias sociales y de la salud: 01 antropóloga, 01 comunicador social, 01 sociólogo, 01 psicólogo, 01 médico psiquiatra y 01 enfermera. Estas seis canciones fueron:

- "Vida bohemia"

- "El borracho"

- "Por tu culpa cervecita"

- "En una cantina”

- "Tragos amargos"

- "Para que quiero la vida".

Una vez elaborado el instrumento de análisis (FICHA DE REGISTRO DE REPRESENTACIONES SOCIALES Y ATRIBUCIONES EN EL CONSUMO DE ALCOHOL EN CANCIONES DE LA SIERRA PERUANA), se procedió a analizar las 
106 canciones tamizadas.

\section{RESULTADOS}

De las 531 canciones revisadas en general como propias de la sierra peruana, el $20 \%$ en sus discursos y contenidos hacen clara y definida alusión al consumo de alcohol y la subvaloración personal que, postulamos son expresiones socio culturales que caracterizan parte la subjetividad de los pobladores de esta gran región del territorio peruano.

Del análisis de las 06 canciones elegidas como representativas, se identificaron 05 categorías de representación social, de los cuales 01 fue para el consumo de alcohol y 04 para la subvaloración personal, y 06 categorías de atribución que a continuación se analizan:

\subsection{Categoría de representación social}

\subsubsection{Categorías de representación sobre el consumo de alcohol}

El licor / alcohol como remedio para las penas y sufrimientos, está presente en el $64.28 \%$ de las canciones referentes al consumo de alcohol, equivalente al $6.77 \%$ del grupo de canciones de la sierra en general. Sus estrofas, por ejemplo, designan al licor de diferente manera, como; “cervecita”, “copas de amor", "licor maldito”, “cervecita negra”, "vaso de cerveza", "trago amargo", "la cantina", "bendito licor", etc.

Estas canciones a través de su discurso lírico dan a entender que en las serranías del Perú, libar bebidas con contenido etílico, no importando el grado de toxicidad es una práctica frecuente en la interacción social o en situaciones diversas de la existencia humana bajo la representación de ser el medio que ayuda y permite se mitiguen o remedien las penas y sufrimientos de cualquier índole, lo que de alguna manera predispone y justifica su consumo en un estilo desenfrenado y de riesgo como: "hasta emborracharse", "hasta más no poder", "hasta no saber quién soy”, "hasta las últimas consecuencias”, "hasta morir”, etc.

En este sentido, por ejemplo, es frecuente escuchar expresiones o títulos canticos como:

- "en el fondo de una copa podré olvidarte" (LLORANDO A MARES)

- “licor maldito bríndame el olvido" (OH LICOR MALDITO)

- “quiero tomar, quierobeberparaolvidarmitriste dolor"(QUIEROTOMAR)

- “en un vaso de cerveza mataré mi tristeza”(CERVECITA, MAS CERVECITA)

- "sólo en las cantinas con tragos amargos me olvido todo un poco de viles engaños, de negras acciones, de ingratitudes" (TRAGOS AMARGOS) 


\subsubsection{Categorías de representación de subvaloración personal}

Autoconcepto negativo con resignación al abandono y sufrimiento, alcanzan al $91 \%$ de las canciones analizadas, donde se identifican una pluralidad de expresiones denotativas, simbolizadas, analogizadas, en metonimias, e incluso en alusiones directas que revelan una condición y representación de sí precarias con pobre autovaloración, marcadas por la desesperanza, fatalismo, inutilidad, minusvalía y tolerante espera a un inevitable sufrimiento.

\section{Expresiones o títulos de canciones que ejemplifican esta forma de representación son:}

- "en mis canciones dejo el recuerdo de mi vida, no me desprecien comprendo que ya nada valgo"(VIDA BOHEMIA)

- “asi es la vida amigas mías, sufrir por tantas decepciones” (QUIENTOMAS MAS QUE YO)

- "en tu vida sólo he sido un juguete que no vale nada” (LA ÚLTIMA COPA)

- "en tu vida seré un martirio, porque yo he nacido sin suerte en esta vida” (AMORAGONIZANTE)

- "para mi ya no hay consuelo, para la mujer desgraciada todo pasa, todo sucede"(MALVACINA)

Evasión con ideas de autoeliminación, enfrentar las demandas de la vida con actitud positiva y constructiva, ameritan en las personas el dominio de un conjunto de habilidades sociales como; buena autoestima, valores, un proyecto de vida con necesidades de logro definidos, capacidad de autocontrol y tolerancia (Alarcón, 2009). Sin embargo, contrariamente el sentido lírico y literal de las canciones motivos de estudio en un $67 \%$ de la muestra de canciones analizadas, que comprende el 13\% del grupo de canciones de la sierra en general, expresan disposición anticipada al abandono con tendencia a evadir y no afrontar los problemas o adversidades.

Expresiones o título de canciones que ilustran esta forma de representación son, por ejemplo:

- “mañana parto hacia lo ignoto” (HASTA SIEMPRE)

- “cuando yo me vaya vivirás dichosa” (PENAS Y SUSPIROS)

- “dime adiós amorcito, a tierras lejanas hoy me retiro" (FALSIA)

- “mañana cuandome vaya lloraras sime has querido”(DE JARANA EN JARANA)

- “como este vientome iré... y nunca regresaré” (MI PALOMITA)

Un importante sub grupo de estas canciones son aquellas que manifiestan ideas de autoeliminación asociados a los deseos de muerte. Representaciones que enuncian esta condición son por ejemplo las siguientes expresiones o títulos cánticos: 
- "en un vaso de cerveza quisiera tomar veneno, veneno para morirme" (EN UN VASO DE CERVEZA)

- “si el engaño es humano venga la muerte” (LLORANDO A MARES)

- "esta vida de bohemia que la muerte se la lleve” (MI VIDA YA NO ES VIDA)

- "al cielo pido/clamo que venga la dicha de la muerte” (AMARGA SUERTE)

- “entregarmividaquisieraalosfilosde uncuchillo”(A LOS FILOS DE UNCUCHILLO)

Demandas de compañía y afecto, el $39.62 \%$ del grupo de canciones analizadas, que comprende al $8 \%$ del grupo de canciones de la sierra en general, expresan representaciones de intolerancia a la soledad con imperiosas demandas de compañía y correspondencia afectiva del ser amado que, de no ser atendidas serían la fuente de perturbación afectiva, emotiva y/o conductual que afectaría el bienestar y al buen desempeño social.

Algunas expresiones canticas y títulos que por ejemplo revelan estas representaciones son:

- "que triste es la vida y amargo el trabajo, pero más amargo es vivir solito" (EL BORRACHO)

- “tu ausencia me trae tristezas, sin tu amor mi vida no vale nada” (TU AUSENCIA)

- "toma mi amor, mi vida yo te brindo con cariño, yo sin tu amor voy a morir, sin tus caricias falleceré” (YO TE AMO)

- "porque te quiero / adoro, porque no puedo vivir sin ti vida mía” (EL ABORRECIDO)

Objetivos de vida imprecisos, la ausencia de visión positiva de futuro y de las necesidades de logro son otras de las representaciones presentes en los cantos de la sierra peruana. El $38 \%$ de las canciones analizadas reflejan en sus enunciados una desorientación existencial, o que todas las perspectivas de desarrollo personal están en función a la persona amada. Esta forma de representación está presente en el $7.53 \%$ del grupo de canciones la sierra peruana en general.

\section{Expresiones canticas y títulos que entrañan esta condición son, por ejemplo:}

- "sola siempre sola, voy rodando por el mundo sin consuelo en la vida" (SOLA SIEMPRE SOLA)

- “el mundo para mí no tiene claridad” (SE VA MI AMOR/MALAYA MI SUERTE)

- “que será pues mi destino, donde estará pues mi suerte”(MI SUERTE)

- “asíhabrá sidomi destino, triste y solo, siempre errante” (PALOMA HERIDA)

- “soycomo aquellas hojas. arrastrado por el viento ya no sédónde caer"(SOY LA MALA YERBA) 


\subsection{Atribuciones para el consumo de alcohol y la subvaloración personal}

Las atribuciones identificadas con respecto al consumo de alcohol y la subvaloración personal fueron seis que, jerárquicamente por su frecuencia se analizan a continuación:

Resentimiento, es un sentimiento incómodo y recurrente por los recuerdos de algún maltrato vivenciado, sea por alguien, un grupo de personas, una institución o por la vida en general. Dicho maltrato impidió el logro de ciertos bienes materiales o espirituales a los que la persona creía tener derecho; en consecuencia, la persona siente violada o negado en el ejercicio del acceso a la justicia y la equidad. tal como lo define (Arroyave (2019, p.97) como "un estado emocional de malestar por el daño que otro individuo o un grupo le infligió a alguien, como cuya característica principal es el rencor por la ofensa recibida por otra persona, que se considera como hostil, enemiga o victimario".

Bajo esta óptica, el elemento del resentimiento como factor que atribuye o induce al consumo de alcohol y la sub valoración personal se ha identificado en el $28.30 \%$ de las canciones analizadas, y equivalente al $5.64 \%$ del grupo de canciones de la serranía peruana en general. Así mismo, se debe precisar que las expresiones de resentimiento identificadas en las canciones no se generan necesariamente por la condición social o de pobreza en se vive, sino que están en relacionadas más bien a frustraciones de tipo amoroso en la relación de pareja, como ejemplifican las siguientes estrofas o títulos:

- ahora que me encuentro triste y afligida, no quiero recordar aquel maldito amor (OH LICOR MALDITO)

- ya no quiero más engaños, porque en tu vida solo he sido un juguete que no vale nada (LA ULTIMA COPA)

- los desprecios de tus padres no olvidaré porque opacaron mi sentimiento y mi gran pasión (EL ABORRECIDO)

- mírame amor ingrato, mírame que triste estoy, después que tanto te quise me pagaste con traición (DESDICHADO AMOR)

Frustraciones afectivo-emocionales, hace referencia a cierta forma de sentimiento de las personas cuando no logran satisfacer un deseo planteado y anhelado. Ante este tipo de situaciones, la persona suele reaccionar con expresiones de ira, ansiedad, incluso disforia. Tal experiencia, se convierte en un motivo que induce a consumir alcohol, o a sub valorarse.

Esta atribución en las canciones analizadas se inculpa más a situaciones de desengaño en 
la relación de pareja como se ejemplifican en las siguientes expresiones canticas:

- "solo en las cantinas, me olvido todo" (TRAGOS AMARGOS)

- “tomaré para olvidar falsas palabras de amor" (TOMO PARA OLVIDARTE)

- “dos cervezas porfavor quiero brindar por ese amor que me engañó, quiero olvidar la traición de su amor" (DOS CERVEZAS)

- "un par de cervezas quisiera tomar, dicen que no valgo para tu querer, mi vida arruinaste con tu traición" (UN PAR DE CERVEZAS)

- "que dolor me estas causando con tus engaños, me emborracho por tu amor, me emborracho"(ME EMBORRACHO POR TU AMOR)

Sufrimiento y desamparo, implica una forma de sensación que genera infelicidad y está presente en toda condición de vida, puede ser por causas físicas, psicológico o afectivas. Cuando la persona ante este sentimiento no recibe ayuda, protección o compañía, se dice está en situación de desamparo. En el grupo de canciones analizadas, se aprecia que el sufrir, sentir desamparado o abandonado por el ser amado en la relación de pareja es una atribución preponderante para consumir licor o subvalorarse.

\section{Expresiones cánticas y títulos en este sentido son, por ejemplo:}

- "tomo para olvidarte y porque quiero calmar mi sufrimiento" (TOMO PARA OLVIDARTE)

- "quiero de muchas maneras dar fin a estas mis penas, para no seguir sufriendo ni sufrir malas voluntades” (EN UN VASO DE CERVEZA)

- "como quieren que no beba si estoy sufriendo, desde el día que te fuiste mi vida no tiene calma, me he entregado a la bebida por tu abandono" (POR TU CULPA)

- "huerfanito soy sin padre ni madre, pobre hay de mi” (DONDE ESTARÁS)

- "hoy para mí no hay consuelo, hasta las flores del campo para mí se han marchitado, es la desdicha que yo tengo" (EL PERFUME DE UNA ROSA)

Enamoramiento, es un estado emocional donde la persona es poderosamente atraída por otra, generándole alegría, comprensión y disposición para compartir la vida con aquella persona. Este estado alcanza niveles de apasionamiento cuando hay deseos impulsivos por un contacto físico en intimidad, deseos profundos de correspondencia, pensamientos rumiantes, nerviosismo y ansiedad ante la persona por quien se es atraído, o ver sólo cualidades en esta persona sin admitir rasgos negativos.

Expresiones y títulos cánticos que connotan esta condición son, por ejemplo: 
- "sumergida en la bebida sufro por quererte lamarte tanto" (MI VIDA YA NO ES VIDA)

- " "porqué,por qué, porqué me enamoréde ti, deti, nadamenos deti, cuantomás borrachita más y más te quiero" (POR QUÉ ME ENAMORE)

- “me emborracho por tu amor, me emborracho" (ME EMBORACHO POR TU AMOR)

- “entre licor y licor pronuncio tu nombre, que destino injusto de quererte tanto" (ENTRE LICOR Y LICOR/PERDONA DIOS MÍO)

Recuerdos y el desconsuelo, se refieren a aquellas reacciones ante la pérdida de un ser querido asociado muchas veces a síntomas físicos o pérdida de energía. Un sentimiento se expresa en desconsuelo cuando se acompaña de angustia, aflicción y convencimiento que las penas y sufrimientos no cesarán o no tendrán alivio.

\section{Expresiones y títulos canticos que connotan esta condición son, por ejemplo:}

- "desgarrada por los tragos y el desconsuelo recordaré de tu nombre” (LLORANDOA MARES)

- "él se fue dejándome sola, recuerdos imborrables para olvidar” (TOMARÉ PARA OLVIDAR)

- “yo ya no puedo borrarlo de mi mente, es el culpable de mi sufrimiento” (POR QUÉ DIOS MÍO)

- "quisiera matarme para olvidarte porque es imposible dejar de quererte, no puedo olvidarte” (MATARME QUISIERA)

- "quisiera olvidarte, pero no he podido, este amor maldito mendiga me tiene" (QUISIERA OLVIDARTE)

Dependencia alcohólica, se refiere a una necesidad imperiosa y compulsiva de libar bebidas alcohólicas. El alcohol es una sustancia con poder adictivo, cuya dependencia se categoriza como el "Síndrome de dependencia del alcohol” (AAP - DSM-5, 2013) que, se le conoce como alcoholismo.

Las atribuciones para el consumo de alcohol y que se ponen de manifiesto en parte del cancionero de la sierra peruana son; los deseos incontrolables de beber licor, así como las incompetencias para el autocontrol.

Textos y títulos líricos por ejemplo que anuncian de manera directa o indirecta necesidades etílicas, degradación y deterioro en la condición humana debido a dependencia alcohólica son:

- " por tu culpa cervecita, ahora me encuentro abandonado en la miseria y la pobreza" (POR TU 


\section{CULPA CERVECITA)}

- "prometí ya no volver más a beber, sin embargo, más y más estoy tomando" (DOS MÁS)

- "hoy quiero seguir tomando, traigan cerveza y más cerveza” (UNA CERVEZA)

- “a ti cervecita quiero olvidarte y no puedo”(CERVEZA MAS CERVEZA)

- "soyborracho, borrachoperdido, andotomandodecantinaen cantina" (CONVENCIDO ESTOY)

\section{DISCUSIÓN DE LOS RESULTADOS}

Las representaciones sociales para el consumo de alcohol develadas en las canciones son formas difundidas de percepción. En estas representaciones sociales, el beber alcohol es un acto conceptualizado y valorado como el remedio para las penas y el sufrimiento, y en el sentido subjetivo, denotan sentimientos marcados por el fatalismo, inutilidad, resignación al abandono, sufrimiento, ideación suicida, necesidades absolutas de afecto y compañía, en el marco de objetivos de vida imprecisos que, en resumen, dimensionan una pobre o baja autoestima.

En cuanto a atribuciones, como parte de la subjetividad social, al acto de beber alcohol se le atribuyen sentimientos como el resentimiento, frustraciones afectivo-emocionales, sufrimiento, desamparo, enamoramiento, los recuerdos y el desconsuelo, o la dependencia alcohólica en sí misma. Esta condición se corrobora con los hallazgos de Morales (2017), en cuanto a las expectativas de futuro en pobladores que viven en contextos de pobreza en los asentamientos humanos del Perú; resaltan atribuciones para el consumo de bebidas alcohólicas en el sentido que sirven como una forma de desahogo a la desesperanza por carecer de recursos económicos y/o por problemas sentimentales, asociado al recuerdo de experiencias de abandono parental y la ocurrencia de escenas de violencia en el interior del hogar.

En aproximación a estas formas de representación y atribución en referencia al género musical "chicha peruana", desde la mirada sociológica, Hurtado (1995), en el libro “Chicha Peruana” a partir del análisis de contenido de una antología de este género indica que, la presencia y avance de este género evidencia el peso de los migrantes en la cultura urbana y cómo se van transmitiendo ciertos valores de la mentalidad andina y popular en relación a: las raíces andinas, el trabajo, el tema amoroso, el machismo y el alcohol; la

\footnotetext{
${ }^{1}$ Chicha peruana, género musical producto de la fusión del huayno, rock y la cumbia que surge del fenómeno migratorio de la sierra a ciudades de la costa peruana, principalmente de la capital Lima.
} 
religión y el humor; y lo social-político, siendo manifiesto en el discurso cantico las expresiones de sumisión, miseria, la conexión con la delincuencia, la situación de los menores, huérfanos y/o migrantes, la resistencia, la protesta, la búsqueda de la liberación. Todo lleva al autor a postular finalmente que las canciones son expresión del alma de un pueblo, de su identidad y condición, y por lo tanto reflejan - cuando son auténticas también las aspiraciones de vida.

Es el aprendizaje social o vicario, planteados por Bandura y Walters (1990), el mecanismo por el que estas representaciones y atribuciones se adquieren y asimilan, donde las expresiones folclóricas y canticas se convierten en legítimos condicionantes y modeladores de estilos de vida y exteriorización de los sentimientos y expectativas de las individualidades y colectividades. Así mismo, desde la mirada del paradigma de la indefensión aprendida de Rosellini y Seligman (1975) y Seligman (1998), los mensajes de las canciones motivo de análisis, estarían induciendo a entender que la subvaloración personal, el sufrimiento por el abandono, la pobreza, los desengaños y frustraciones son inherentes a la existencia humana, no habiendo otra alternativa más que aceptarlo y resignarse a vivir así.

Escalante (2017), en el análisis sobre el huayno como expresión de la identidad cultural de los pobladores de Huamanga - Ayacucho, advierte que, a través del ritmo, la melodía, el canto y la danza del huayno se transmiten valores culturales a las nuevas generaciones, aspecto eminentemente formador y educativo; sin embargo, no todo se muestra positivamente formativo ya que, las letras de algunas de estas canciones resaltan en demasía mensajes de una cultura de pobreza, atraso, conformismo y sufrimiento, deformando y mancillando la personalidad del habitante andino. El huayno, cantado o bailado en cualquier acontecimiento festivo social o familiar, se convierte en un medio por el que los pobladores andinos, de la serranía peruana se sensibilizan, concientizan y afirman su identidad.

La construcción de una identidad social deviene como resultado de la interacción entre procesos de naturaleza cognitiva, evaluativa y emocional que funciona como una instancia articuladora entre el comportamiento individual, la cognición social y diversos fenómenos colectivos (Hogg y Ridgeway, 2003) donde, consideramos son ineludibles las representaciones y atribuciones en tanto, implican una aproximación al entendimiento de un rasgo social preponderante de los grupos o las colectividades humanas (Espinosa y 
Tapia, 2011).

\section{CONSIDERACIONES FINALES}

Los debates económico-sociales y culturales reconocen a muchos pueblos de la sierra peruana como "Los más pobres del Perú", calificación no muy lejana de la realidad. Al respecto, es válido preguntarse: ¿Las representaciones sociales y atribuciones develadas en canciones como las que han sido analizadas expresan una subjetividad de pobreza?, ¿Las políticas públicas de salud y desarrollo humano estarán tomando en cuenta el factor de riesgo que implica la difusión del cancionero que alude al licor y la subvaloración personal?, o en el sentido de la trascendencia, ¿Cuáles serán los motivos e ideales que impulsan a vencer y superar cuanto obstáculo se tenga como personas, familia y sociedad?, ¿Qué fines supremos se convierten en la razón de la existencia y a qué decisiones orientan los objetivos de vida?, ¿Cuál debería ser el rol de los artistas musicales o canta autores, como agentes formadores de la identidad y subjetividad social de los pueblos?.

Consideramos que estas representaciones y atribuciones develadas en el discurso lírico del grupo de canciones analizadas, lejos de ser una fortaleza en su identidad social y cultural, son debilidades cuyos efectos e influencias no son favorables para el bienestar de las personas, la familia y la sociedad que, no siempre en el Perú, este tipo de desvalores están siendo abordados por la educación formal y las políticas sociales, comunicacionales y de desarrollo humano. Muchos de los contenidos canticos, al ser difundidos, directa o indirectamente promueven estilos de vida de riesgo. Resulta necesario que, a partir del análisis y entendimiento de los factores expuestos se propongan estrategias de intervención, capaces de contribuir a la formación de una cultura saludable, pacífica, solidaria y de convivencia armoniosa como base para el desarrollo integral de los pueblos de la serranía peruana, en tanto sus carencias y problemática no sólo se circunscriben única y exclusivamente a lo económico, sino también a lo sociocultural y psicosocial.

Son algunos los artistas musicales o canta autores, lejos de ser agentes sociales de educación y referentes positivos ante la comunidad, en un sentido de apología, pregonan bondades al alcohol como el remedio de las penas y sufrimientos. Así estos importantes actores sociales desvirtúan o se alejan de un rol formativo, orientador y educador y, por el contrario, estos artistas estarían distorsionando la ética, la moral, los derechos, las responsabilidades, los deberes, y los comportamientos saludables.

Finalmente, existe una intrínseca reciprocidad entre el bienestar personal y el bienestar 
social que prácticamente son procesos psicológicos interdependientes. En tal sentido, las representaciones sociales y las atribuciones resultan ser muy importantes no sólo en relación al consumo de alcohol o la subvaloración personal, sino en general respecto a todas las diversas manifestaciones de comportamiento humano ya sean de riesgo o protectoras. En particular en las expresiones de riesgo, es necesario, por ejemplo, analizar cómo afectan o influyen los factores psicosociales y de identidad en fenómenos como, las adicciones, la violencia en todas sus formas, los estilos de vida, la corrupción, delincuencia, etc. La respuesta es que los factores psicosociales y de identidad son determinantes, pudiendo llegar a limitar o frenar las capacidades de los individuos para afrontar a los problemas y adversidades de la vida.

Las personas cuyos constructos psicosociales y de cognición social están conformadas por valores positivos, serán más proclives a construir generacionalmente su bienestar. El bienestar social coherente y sostenible permite evaluar la relación directa con el bienestar personal, (Cicognani et.al., 2008) o, como alegan Park, Peterson y Sun (2013), expresar y conceptualizar la vida en un sentido optimista es muy beneficioso porque se asocia a una disposición para afrontar y resolver los problemas de manera activa. Dicha disposición psicológica positiva permite que se potencien las cualidades personales, sociales y culturales en favor de la superación de las dificultades. Ello, en las explicaciones y conclusión de Seligman (1998), implica que el carácter optimista o pesimista dependerá de la interpretación que las personas den a los hechos que les ocurran en el transcurrir de sus vidas, y del sentido de responsabilidad con el que la asumen.

\section{REFERENCIAS BIBLIOGRAFICAS}

AAP - Asociación Americana de Psiquiatría (2013), Guía de consulta de los criterios diagnósticos del DSM 5. Arlington, VA, Asociación Americana de Psiquiatría. D.F: American Psychiatric Publishing.

file://C:/Users/HP/Desktop/REVISTA\%20UNMSM/dsm-v-guia-consulta-manualdiagnostico-estadistico-trastornos-mentales.pdf

Alarcón, R. (2009). Psicología de la felicidad. Editorial Universitaria, Universidad Ricardo Palma.

Arroyave, O. (2019). Resentimiento como respuesta emocional al daño en dos grupos de mujeres en el conflicto armado en Colombia. Revista de Psicología Universidad de Antioquia, 11(1), 93-115. 
DOI: https://doi.org/10.17533/udea.rp.v11n1a04

Bandura A., Walters R. (1990). Aprendizaje social y el desarrollo de la personalidad. Alianza Universidad.

Cicognani, E., Pirini, C., Keyes, C., Joshanloo, M., Rostani, R y Nosratabadi, M. (2008).

Social Participation, Sense of Community and Social Well Being. A Study 86 on American, Italian and Iranian University Students. Social Indicators Research. 89, $97-$ 112

https://www.jstor.org/stable/27734730

Díaz, J., Pérez, A. \& Florido, R. (2011). Impacto de las tecnologías de la información y las comunicaciones (TIC) para disminuir la brecha digital en la sociedad actual. Cultivos Tropicales, 32(1), 81-90.

http://scielo.sld.cu/scielo.php?script=sci_arttext\&pid=S0258-

$59362011000100009 \& \operatorname{lng}=$ es\&tlng=es.

Escalante, G. El huayno como expresión de la identidad cultural de los pobladores de

Huamanga - Ayacucho, 2017. [Tesis de licenciatura- Universidad Nacional de

San Agustín de Arequipa]. Repositorio Institucional UNSA.

http://repositorio.unsa.edu.pe/handle/UNSA/6218

Espinosa, A. \& Tapia, G. (2011). Identidad nacional como fuente de bienestar subjetivo y social. Boletín de Psicología, 102, 71-87.

https://www.uv.es/seoane/boletin/previos/N102-5.pdf

Heider, F. (1958). The psychology of interpersonal relations. Wiley.

Hirt, E. y McCrea, S. (2002). Cognición social. En Kimble, Ch. et. al. (Eds): Psicología social de las américas. (pp. 91-134). Pearson.

Hogg, M.A y Ridgeway, C. (2003) Social identity: Sociological and social psychological Perspectives. Social Psychology Quarterly, 66, 97-100.

Hurtado, W. (1995). Chicha peruana, Lima. Grupo de Investigaciones Económicas ECO. INEI (2017). Perfil Socio demográfico, Informe Nacional. Censos Nacionales 2017: XII de población, VII de Vivienda y III de Comunidades Indígenas. Instituto Nacional de Estadística e Informática - Perú.

https://www.inei.gob.pe/media/MenuRecursivo/publicaciones_digitales/Est/Lib1539/

INEI (2021). Evolución de la Pobreza 2009-2020. Informe técnico. Instituto Nacional de Estadística e Informática - Perú. 
https://www.inei.gob.pe/media/MenuRecursivo/publicaciones_digitales/Est/pobreza2 020/Pobreza2020.pdf

Jodelet, D. (1993). La representación social: fenómenos, concepto y teoría. En: Moscovici, S. (comp.). Psicología social II. Pensamiento y vida social. Psicología social y problemas sociales. Paidós.

Morales, L. (2020). Expectativas de futuro en pobladores que viven en situación de pobreza de tres asentamientos humanos en Chimbote, Puno y Pucallpa-2017. [Tesis doctoral - Universidad Peruana Cayetano Heredia]. Repositorio Institucional UPCH. https://repositorio.upch.edu.pe/bitstream/handle/20.500.12866/8613/Expectativas_M oralesLopez_Luis.pdf?sequence $=1 \&$ isAllowed $=\mathrm{y}$

Moscovici, S. (2002). Social representations. Explorations in Social Psychology. Polity Press.

Park, N., Peterson, Ch. y Sun, J. (2013). La Psicología Positiva: Investigación y aplicaciones. Universidad de Michigan, Terapia psicológica. Vol. 31, № 1, 11-19 file:///C:/Users/HP/Downloads/La_Psicologia_Positiva_Investigacion_y_aplicacione. pdf

Robayo, M. (2015). La canción social como expresión de inconformismo social y político en el siglo XX. Calle 14, 11(16) pp. 54-67 https://www.redalyc.org/pdf/2790/279042458005.pdf

Rosellini, A., Seligman M. (1975). Frustration and learned helplessness. Journal of Experimental Psychology. Animal Behavior Processes. Vol 1(2), 149-157.

Seligman M.E.P. (1998). Learned optimism: How to change your mind and your life. (2a. ed.). Pocket Books. 